\title{
A New Energy Device for Skin Activation to Acute Wound Using Cold Atmospheric Pressure Plasma: A Randomized Controlled Clinical Trial
}

\author{
Akio Nishijima $^{1-3 *}$, Takahiro Fujimoto ${ }^{2,3}$, Takamichi Hirata ${ }^{3}$ and Junko Nishijima ${ }^{2,3}$ \\ ${ }^{1}$ Department of Plastic and Reconstructive Surgery, Japan \\ ${ }^{2}$ Clinic F, Japan
}

${ }^{3}$ Department of Medical Engineering, Japan

*Corresponding author: Akio Nishijima, Department of plastic and reconstructive surgery, University of Tsukuba, 2-1-1

Amakubo, Tsukuba-shi, Ibaraki, Japan

ARTICLE INFO

Received: 幽 August 21, 2019

Published: 㓞 August 28, 2019

Citation: Akio Nishijima, Takahiro Fujimoto, Takamichi Hirata, Junko Nishijima. JilingZhong. A New Energy Device for Skin Activation to AcuteWound Using Cold Atmospheric Pressure Plasma: ARandomized Controlled Clinical Trial. Biomed J Sci \& Tech Res 21(1)-2019. BJSTR. MS.ID.003532.

Keywords: Cold Atmospheric Pressure Plasma; Plasma Medicine; New Energy Device; Wound Healing; Skin Activation

\section{ABSTRACT}

Objectives: The aim of this study was to evaluate Cold Atmospheric Pressure Plasma (CAP) treatment as a novel energy device for skin activation, assisting its antiinflammatory action and promoting wound healing.

Materials and Methods: This study was a prospective, randomized, open, blindedendpoint method and controlled trial conducted on the forearms. This study was conducted on 48 regions of 12 healthy volunteer subjects. A fractional CO2 laser was used to irradiate four similarly sized regions on the left forearm. Each region then randomly received one of the following treatments: plasma (radiated with a CAP jet for 60 seconds), betamethasone valerate and gentamicin sulfate, basic fibroblast growth factor, or no treatment. A three-dimensional dermal analysis device was used to measure the $\mathrm{a}^{*}$ index $\left(\mathrm{a}^{*} \mathrm{I}\right)$, arithmetical mean roughness $(\mathrm{Ra})$, and average melanin density immediately before and after the laser radiation, and at post-treatment Days 1, 3, 7, 14, and 28.

Results: The day after therapy, a*I of the plasma group was significantly improved compared with the control group $(p=0.03)$. However, from Day 3 , these two groups showed similar tendencies (with no significant differences) in all evaluation items. No complications of pain, infections, or bleeding were observed in any treated regions after laser radiation.

Conclusion: In this study, anti-inflammatory action by the CAP radiation were observed, obtaining a treatment efficacy equivalent to that of conventional therapies. Our findings provide evidence of the effectiveness and safety of CAP and assist the future development of medical plasma therapy.

\section{Introduction}

More than a half-century has passed since Maiman's first success with ruby laser treatment 1 [1-4]. Since then, laser devices have been developed for use in diagnosis and treatment across several fields of medical practice, including those for skin activation. Various energy devices can be used for skin activation, as determined by selective photothermolysis theory. Those currently used with demonstrated clinical efficacy include pulsed lasers 2 , fractional lasers 3 , radiofrequency devices 4 , high-intensity focused ultrasound [5], and selective cryolipolysis [6]. In recent years, plasma treatment to promote skin activation has also been attracting attention, with a rapid increase in interest in medical devices based on Cold Atmospheric Pressure Plasma (CAP). Laroussi first reported the use of atmospheric pressure plasma in 1996 [7]; since then, CAP has been investigated for various biomedical applications using appropriate laboratory testing. Skin activation, such as wound healing, is of particular interest with promising studies demonstrating the benefits of [8]. 
Our study used Three-Dimensional (3D) dermal analysis equipment in a randomized controlled trial to evaluate the effect of CAP treatment for skin activation during the process of wound healing of acute wounds in humans. We also compared the efficacy of CAP treatment with that of conventional treatments for skin activation.

\section{Material and Methods}

\section{Study Design}

This prospective, randomized, open, blinded-endpoint method and controlled trial was conducted from September 2017 to April 2018 (University Hospital Medical Information Network identifier: UMIN000029121) comparing treatments on four similarly sized regions on the left forearms of healthy volunteer subjects. The study protocol was approved by the institutional review board of Tokyo City University (IRB number: 413), and all the subjects provided written informed consent.

\section{Subjects}

Initially, the study enrolled 15 healthy volunteer subjects (12 women and 3 men) with a mean age of $36.8 \pm 12.8$ years and Fitzpatrick skin type III or IV, all recruited at the Tokyo City University and Clinic F in metropolitan Tokyo. However, 3 subjects were unable to attend the follow-up appointments regularly, so 12 subjects were included in the analysis. They wore long sleeves to protect their skin from solar radiation. Subjects were excluded if they felt unwilling to be assigned to any of the approaches.

\section{Creation of Lesions by Randomization}

The 12 subjects each received laser treatment to four areas of the same size $(1.5 \times 2.0 \mathrm{~cm})$ on the medial side of the left forearm, making a total of 48 treatment regions. Each lesion was created using a fractional $\mathrm{CO}_{2}\left(\mathrm{FXCO}_{2}\right)$ laser therapy device (SmartXide

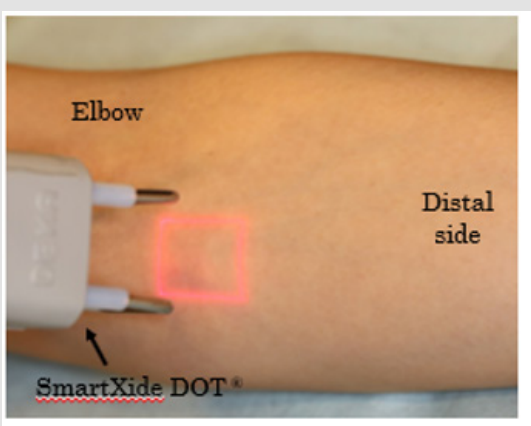

(A)
DOT RTOR, DEKA M.E.L.A. srl, Calenzano, Italy) in a single shot at an output power of $10 \mathrm{~W}$, pulse width of $600 \mu \mathrm{s}$, dot spacing of 650 $\mu \mathrm{m}$, and stack 2 .

\section{Intervention}

After $\mathrm{FXCO}_{2}$ laser therapy, each of the four regions on their arms was subjected to a different treatment: CAP (the CAP group of regions), steroid and antibiotic ointment (ointment group), basic fibroblast growth factor (bFGF group), or no treatment (control group). These interventions were applied only once and were randomly allocated to the regions. The CAP group of regions was subjected to a CAP jet (described below), in accordance with the previously reported laser radiation method for clinical practice $[9,10]$. The ointment group received a topical application of ointment containing betamethasone valerate and gentamicin sulfate (Rinderon-VG®, Shionogi \& Co., Ltd, Tokyo, Japan). The bFGF group received two to three sprays of aerosolized trafermin (genetical recombination) (Fiblast ${ }^{\circledR}$ Spray, Kaken Pharmaceutical Co., Ltd, Tokyo, Japan).

\section{CAP Treatment}

Plasma was applied to the target region with CAP jets using a kINPen MED® (INP Greifswald/neoplas tools GmbH, Greifswald, Germany) [11]. The device used in this study fulfilled the technical requirements for medical use and consisted of a hand-held unit for the generation of gas discharge under atmospheric pressure conditions and a direct current power supply unit. A high voltage of 2-3 kVpp at a frequency of $1 \mathrm{MHz}$ was periodically applied to the pin electrode (frep $=2.5 \mathrm{kHz}$, plasma duty cycle $=1: 1$ ). The system was operated with argon gas at flow rates between 4 and $6 \mathrm{~L} / \mathrm{m}$. Under these working conditions, the plasma jet outside the ceramic capillary was approximately $10 \mathrm{~mm}$ long. Plasma treatment for the target area was performed by moving the visible tip of the plasma jet repeatedly over the whole target area for 60 seconds Figure 1.

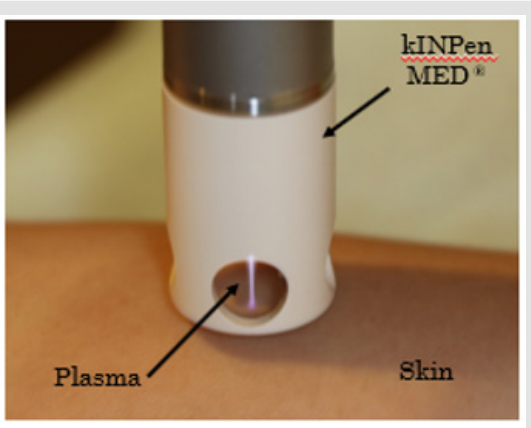

(B)

Figure 1: Fractional $\mathrm{CO}_{2}$ laser $\left(\mathrm{FXCO}_{2}\right)$ and Cold Atmospheric Pressure Plasma (CAP);

(A) $\mathrm{FXCO}_{2}$ laser radiation on the medial surface of the left forearm using a SmartXide DOT RT®.

(B) CAP therapy using a kINPen MED® cold plasma device.

\section{Photographic Analysis}

Before and after the treatment, a 3D skin analyzer (ANTERA 3DTM, Miravex Co., Ltd, Ireland) was used to record changes in the irradiated areas and to assess the process of wound healing [12,13].

\section{Outcome Measurement Methods}

To evaluate the process of wound healing, redness, roughness, and pigmentation on the skin surface were measured. The $\mathrm{a}^{*}$ index (a*I) of $\mathrm{L}^{*} \mathrm{a}^{*} \mathrm{~b}$ color space was used to evaluate redness, with higher 
values of a*I am indicating more red/violet components and smaller values indicating more blue/green components. The roughness of the skin was evaluated by the Arithmetical Mean Roughness (Ra), presented as the average of the absolute values within the sampling region. Pigmentation was evaluated as the mean melanin concentration, as shown by the ANTERA 3DTM. We established a quantitative evaluation method to assess the rate of change in the treated regions. In general, the condition of a wound is influenced by the patient's condition and by environmental factors at the time of monitoring the wound. The quantitative evaluation of the skin condition in the present study, therefore, required the treatment regions to be compared with the intact skin near the radiated sites. The mean values of each evaluation measure for each entire treated region before and after the treatment were obtained from the ANTERA 3DTM image data, and the changes were calculated by comparing the data of the laser-radiated regions and the intact skin. The rate of change (\%) was calculated by the following equation as shown in Figure 2, where $\mathrm{A}$ is the ratio of $\mathrm{a}^{*} \mathrm{I}$ in the radiated lesion relative to the intact skin before the randomized treatment, and $\mathrm{B}$ is that after the randomized treatment:

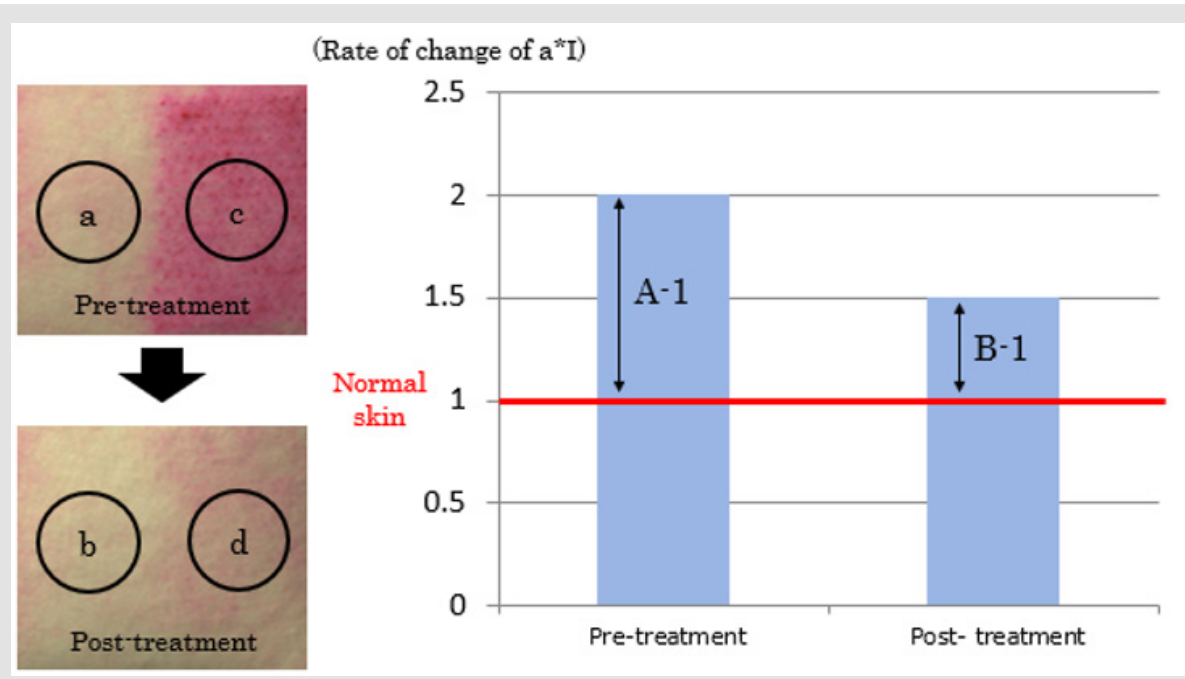

Figure 2: Definition Of rate of change in this study.

Normal intact skin ( $a$ and $b$ ) and regions received treatment (c and d) are plotted.

$\mathrm{A}$ (measured ratio before treatment) $=\mathrm{c} / \mathrm{a}$

$B$ (measured ratio after treatment) $=b / d$

Rate of change $(\%)=(B-1) /(A-1) \times 100$

$(B-1) /(A-1) \times 100$

Improvement was defined when a rate of change was less than $100 \%$.

The evaluation items were measured immediately before and after the $\mathrm{FXCO}_{2}$ laser radiation, and at 1, 3, 7, 14, and 28 days after the radiation. The primary outcome was the change in absolute values and the rates of change of $\mathrm{a}^{*} \mathrm{I}$ and Ra. The secondary outcome was the extent of pigmentation and the safety of the CAP jet treatment at one-month post-treatment. The measurements were made in a blinded manner by a different clinician from the one who performed the treatments.

\section{Statistical Analysis}

No data from any of the 12 subjects were excluded from any analyses. Categorical data were summarized as the frequency and continuous data as the mean and Standard Deviation (SD).
Comparisons between groups were performed using Student's t-test or one-way analysis of variance for continuous variables. The sample size was defined as $4 \times 4$, based on the Latin square method, because we conducted a randomized study for four regions for each subject. The minimum necessary sample number was determined to be 48 sites in 12 subjects, considering collectible number of data in the institutions. On the basis that some subjects may withdraw during the follow-up period, 15 subjects were initially enrolled in this study. All statistical analyses were performed with SAS 9.4 (SAS Institute, Cary, NC, USA). A p-value $<0.05$ was considered statistically significant.

\section{Results}

\section{Flow of Subject Inclusion}

The 12 subjects received treatments after randomizing the selection of treatments, and the outcomes were monitored during the follow-up period Figure 3. 


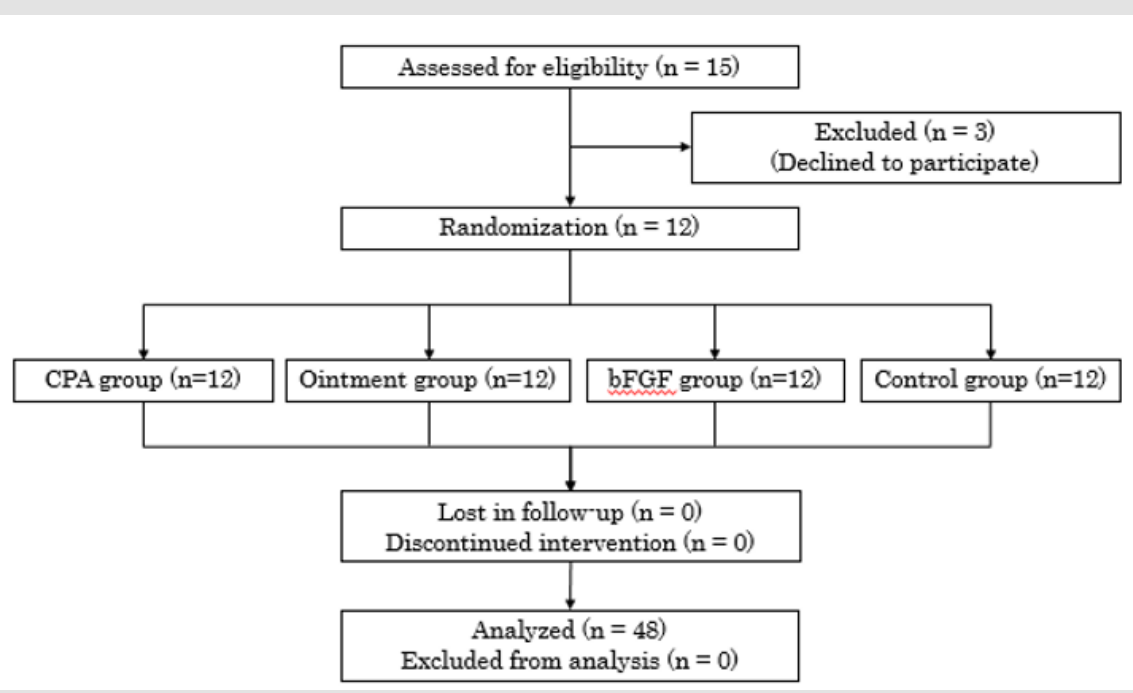

Figure 3: CONSORT flow chart of subject inclusion in our study.

\section{Primary Outcomes}

CAP group vs. Control Group: Figure 4 shows the time course of a*I for the CAP and control groups of treated regions. On the first day following treatment (Day 1), there was a deterioration in $\mathrm{a}^{*} \mathrm{I}$ in the control group, but an improvement in the CAP group. Figure 5 shows the time course of Ra for the CAP and control groups. (Table
1) presents the absolute values and rates of change of a*I and Ra in the CAP and control groups on Day 1 . The results for a*I showed a significant improvement in the CAP group $(p=0.03)$. Figure $6 \mathrm{~A}$ shows representative findings on the medial surface of the left forearm immediately after $\mathrm{FXCO}_{2}$ laser radiation, Figure 6B shows the time courses of $\mathrm{a}^{*} \mathrm{I}, \mathrm{Ra}$, and melanin concentration of the CAP and control groups by visualization using ANTERA 3DTM.

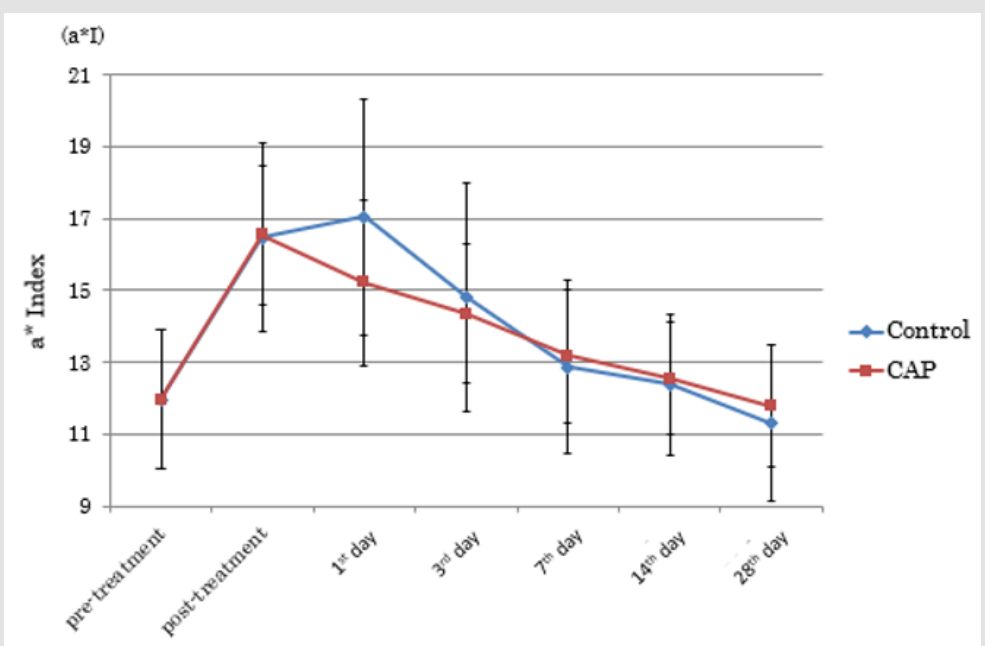

Figure 4: Transition of $a^{*}$ Index in the CPA group and control group.

Note: At 1st post-treatment day, a*I of the control group worsened, but that of CAP group improved.

$(\mathrm{CAP}=$ Cold Atmospheric Pressure Plasma)

Table 1: Absolute values and rate of change of $\mathrm{a}^{*} \mathrm{I}$ and Ra in the control group and the CAP group on $1^{\text {st }}$ post-treatment day.

\begin{tabular}{|c|c|c|c|}
\hline & $\begin{array}{c}\text { Control group } \\
(\mathbf{n}=\mathbf{1 2})\end{array}$ & $\begin{array}{c}\text { CAP group } \\
(\mathbf{n = 1 2})\end{array}$ & $\begin{array}{c}\boldsymbol{p} \text {-value } \\
\mathbf{( t} \text {-test) }\end{array}$ \\
\hline Mean $\mathrm{A}^{*} \mathrm{I} \pm \mathrm{SD}$ & $17 \pm 3.3$ & $15.2 \pm 2.3$ & 0.13 \\
\hline Change rate of a*I \pm SD (\%) & $115.7 \pm 12.3$ & $85.3 \pm 37.2$ & 0.03 \\
\hline Mean Ra \pm SD & $3.2 \pm 0.6$ & $3 \pm 0.8$ & 0.7 \\
\hline Change rate of $\mathrm{Ra} \pm \mathrm{SD}(\%)$ & $62.8 \pm 46.1$ & $48.8 \pm 43$ & 0.47 \\
\hline
\end{tabular}

Note: a*I showed significant improvement in the CAP group.

Abbreviations: $a * I, a *$ index of of L*a*b color space; Ra, arithmetical mean roughness; CAP, Cold Atmospheric Pressure Plasma, SD, standard deviation: 


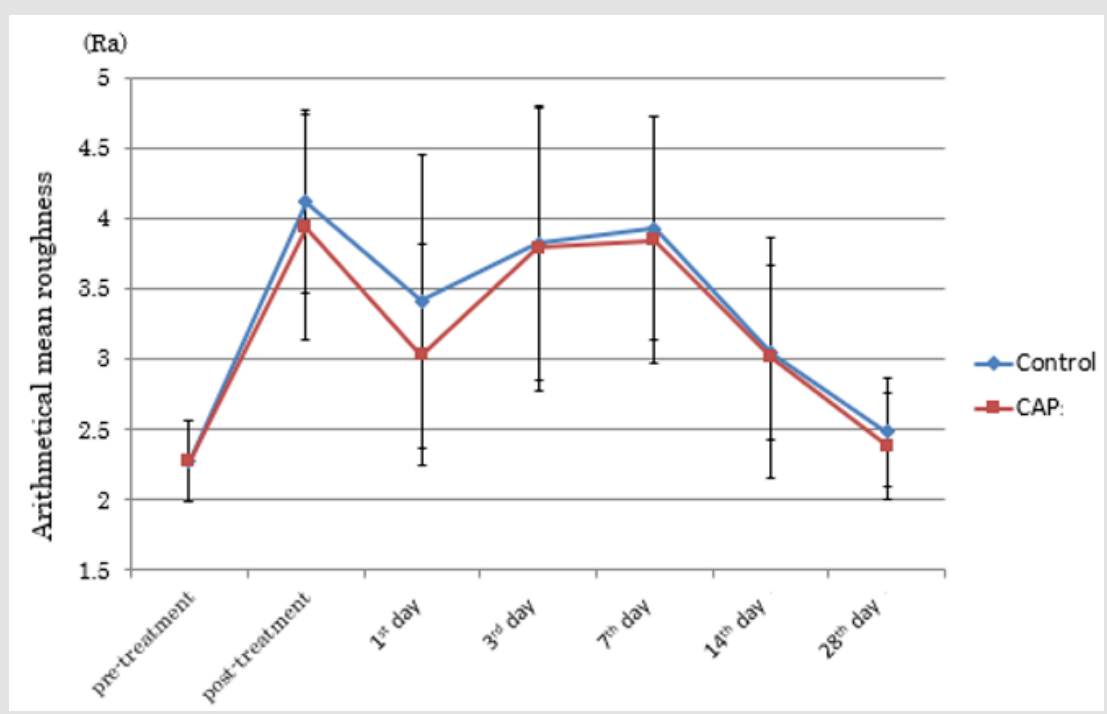

Figure 5: Transition of Ra in the CPA group and control group.

Note: At 1st post-treatment day, Arithmetical Mean Roughness (Ra) of both groups reduced, then increased till $7^{\text {th }}$ posttreatment day. At $28^{\text {th }}$ post-treatment day, both groups showed almost equivalent Ra compared with that before treatment. $(\mathrm{CAP}=$ cold atmospheric pressure plasma)

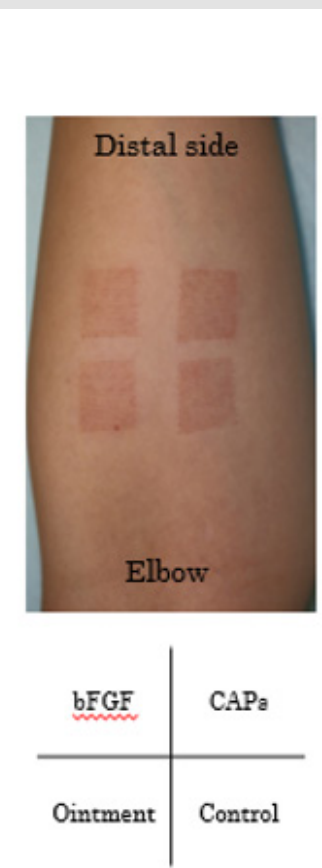

(A)

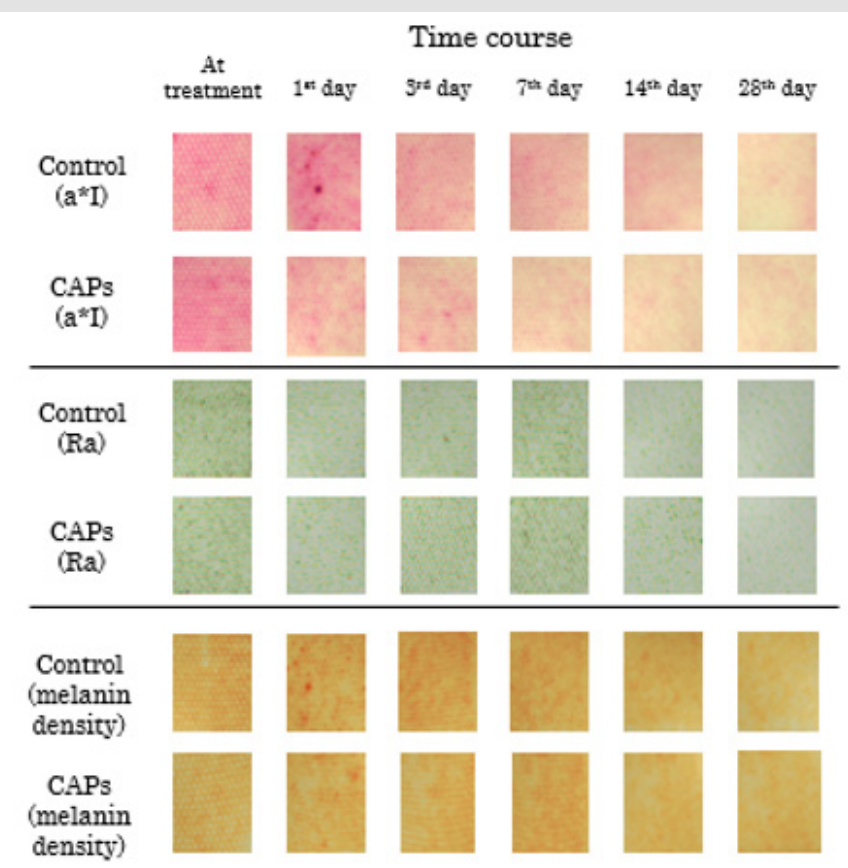

(B)

Figure 6: Evaluation of wound healing;

(A) Findings immediately after $\mathrm{FXCO}_{2}$ laser radiation to the medial surface of the left lower forearm and a randomized example of four-type treatments.

(B) Transitions of a*I, Ra, and melanin concentration of the CAP group and control group, which were visualized by a 3D skin analyzer (ANTERA 3DTM).

CAP, Ointment, and bFGF Groups: Table 2 shows a*I and Ra for the three treatment groups. No significant differences were observed between the CAP, ointment, and bFGF groups at any time point. Also, there were no significant differences in the rates of change. 
Table 2: $\mathrm{a}^{*}$ I and Ra in 3 treatment groups (CAP, ointment, and bFGF).

\begin{tabular}{|c|c|c|c|c|c|c|c|c|c|}
\hline & & \multicolumn{4}{|c|}{$\mathbf{a}^{*} \mathbf{I}(M e a n \pm$ SD) } & \multicolumn{4}{c|}{ Ra (Mean \pm SD) } \\
\hline Time & $\mathbf{n}$ & CAP & Ointment & bFGF & p-value & CAP & Ointment & bFGF & $p$-value \\
\hline Immediately after treatment & 20 & $16.5 \pm 1.9$ & $17.0 \pm 1.6$ & $16.5 \pm 2.3$ & 0.78 & $3.9 \pm 0.8$ & $3.8 \pm 0.8$ & $4.1 \pm 0.7$ & 0.58 \\
\hline $1^{\text {st }}$ day & 20 & $15.2 \pm 2.3$ & $14.8 \pm 2.1$ & $16.5 \pm 3.5$ & 0.3 & $3.0 \pm 0.8$ & $2.9 \pm 0.6$ & $3.1 \pm 0.6$ & 0.81 \\
\hline $3^{\text {rd }}$ day & 20 & $14.3 \pm 1.9$ & $13.7 \pm 1.6$ & $14.0 \pm 3.2$ & 0.82 & $3.8 \pm 1.0$ & $3.5 \pm 0.8$ & $3.9 \pm 1.1$ & 0.78 \\
\hline $7^{\text {th }}$ day & 20 & $13.2 \pm 1.9$ & $12.5 \pm 1.2$ & $12.8 \pm 2.2$ & 0.69 & $3.0 \pm 0.9$ & $3.7 \pm 0.6$ & $4.0 \pm 0.8$ & 0.6 \\
\hline $14^{\text {th }}$ day & 20 & $12.5 \pm 1.6$ & $12.4 \pm 0.9$ & $12.3 \pm 1.6$ & 0.93 & $3.0 \pm 0.9$ & $2.8 \pm 0.7$ & $3.0 \pm 0.7$ & 0.71 \\
\hline $2^{\text {th }}$ day & 20 & $11.8 \pm 1.7$ & $11.5 \pm 0.9$ & $11.5 \pm 2.2$ & 0.90 & $2.4 \pm 0.4$ & $2.4 \pm 0.4$ & $2.4 \pm 0.4$ & 0.95 \\
\hline
\end{tabular}

\section{Secondary Outcomes}

The mean melanin concentrations at Day 28 in the control, CAP, ointment, and bFGF groups were $0.52 \pm 0.06,0.52 \pm 0.07,0.50 \pm$ 0.04 , and $0.53 \pm 0.06$, respectively, with no significant differences between the groups $(\mathrm{p}=0.81)$. No complications, including pain, infection, or bleeding, were observed in any treated region.

\section{Discussion}

\section{Overview of Plasma Devices for Skin Activation}

In 1928, plasma was defined by Irving Langmuir as a state in which there is electrolytic dissociation of the molecules that comprise a gas, where the cations of the molecules and electrons are moving. Arsonvalization was one of the most convenient of the popular electromedical treatments, classified as high-frequency therapy; it had much in common with modern CAP treatment, at least regarding its bioactive properties [14,15]. Jacques-Arsène d'Arsonval discovered the possibility of influencing the human body with high frequencies delivered by his apparatus with the help of extremely high transformation of electric tension [16]. The device was further developed in Germany for a caloric treatment of patients called diathermy, and Rumpf et al. developed a device that differed from the French ones by implementing a capacitively coupled electrode consisting of a Leydener bottle, which was directly applied to the patient's skin [16]. This device can be considered the first plasma source in medicine to use a dielectric electrode.

These days, continuous radiation to the skin is technically possible by using CAP. At a reduced cost and without the need for vacuum equipment, CAP allows the target object to be directly processed with plasma. The kINPen MED device used in this study was the first CAP jet apparatus to receive accreditation worldwide as a medical device (class IIa). Its reusable argon plasma jet can generate a constant, non-thermal (room temperature) plasma at atmospheric pressure [15].

\section{Effect of CAP as a Novel Energy Device for Skin Activation}

Medical Plasma has Two major Effects: Antibacterial action and tissue activation. In clinical practice, the antibacterial action of medical plasma has been attracting attention, especially for the treatment of chronic ulcers such as venous stasis skin ulcers or diabetic foot gangrene [8]. Many types of bacteria and fungi that are categorized as physiological or pathological skin flora are highly susceptible to plasma treatment [17].

In this study, we focused on medical plasma's biological tissue activation effects in acute phase wounds. The significant improvement in redness $\left(\mathrm{a}^{*} \mathrm{I}\right)$ in the regions treated with CAP compared with the control regions demonstrated the antiinflammatory effect of plasma. The process of wound healing is characterized by four continuous, overlapping, and precisely programmed phases:

1) Hemostasis,

2) Inflammation,

3) Proliferation, and

4) Remodeling $[18,19]$.

During all these phases, plasma-derived activation species activate growth factors. Plasma contains many neutral molecules, ion species, and radical species. Nitric oxides are generated when the atmosphere is used as a source for plasma. In 1987, NO was found to play a major part in endothelium-derived relaxing factor, which controls blood flow; blood flow improves after plasma radiation [20]. Hirata et al. reported increased neovascularity with plasma radiation and concluded that the surface reaction of tissue to an electrochemical stimulus activated growth factors and accelerated healing in a gas phase because of the generation of active oxygen, such as Ozone $\left(\mathrm{O}_{3}\right)$ and Hydroxyl radical $(\mathrm{OH})$ and active nitrogen, such as $\mathrm{NO}$ and nitrogen dioxide $\left(\mathrm{NO}_{2}\right)$ [21]. Immediately after the $\mathrm{FXCO}_{2}$ laser treatment, the $\mathrm{Ra}$ of the irradiated skin increased because of swelling. Although no significant differences of Ra were noted between the CAP group and control group, the rate of Ra reduction was greater in the CAP group the day after radiation, indicating a faster reduction in swelling. So, if the wound is more severe than this case, the effects of CAP would be expected to be greater.

The excessive generation of NO carries a risk of inducing abnormality in tissues. We, therefore, evaluated whether activating species caused pigmentation in the skin by measuring CAP-induced melanin concentrations in the irradiated sites. By Day 28, the subjects' skin color had returned to that before laser radiation, 
indicating that the radiation parameters in our study were not a problem. Metelmann et al. monitored radiated lesions for 12 months and reported no delayed side effects, such as carcinogenesis or hypertrophic cicatrization 9. Figure 7 shows the relationship between the activating species generated after plasma radiation and skin activation, based on our study findings.

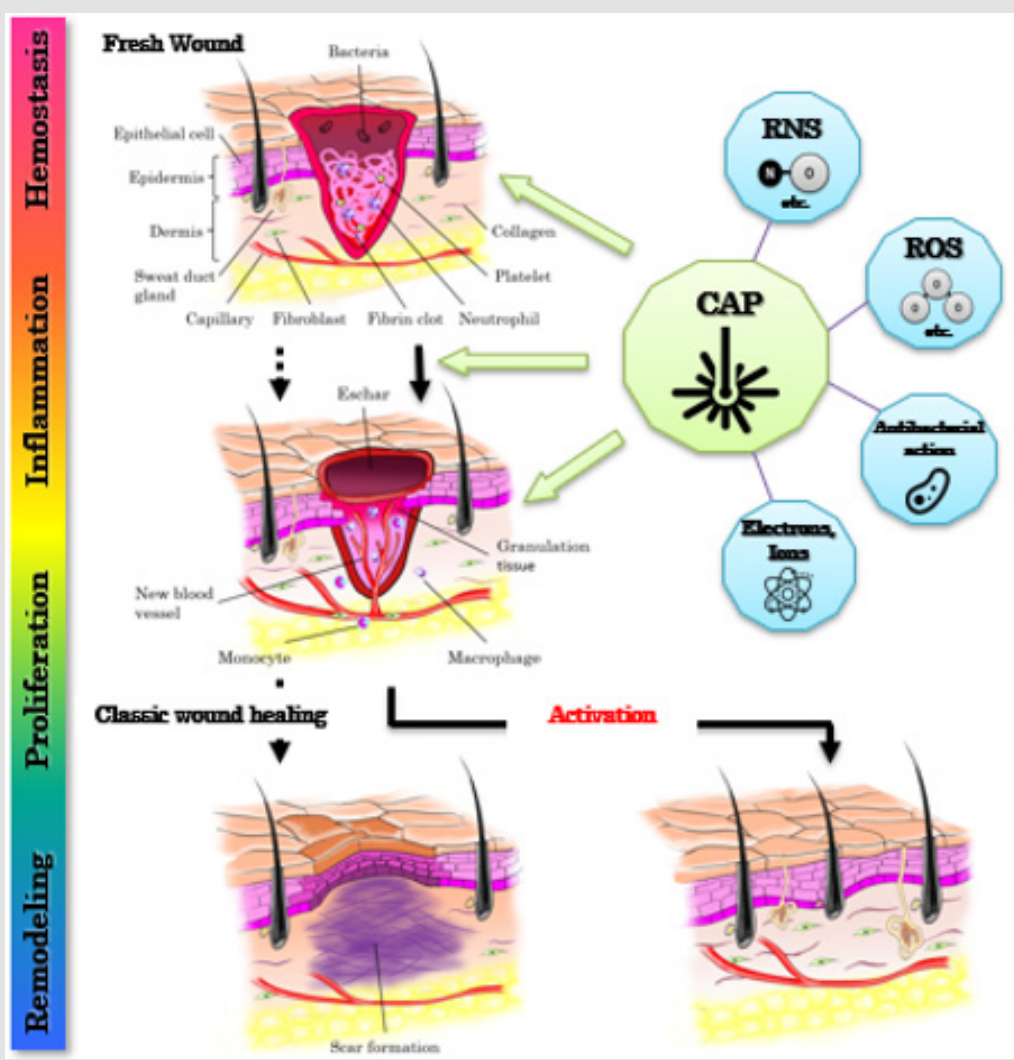

Figure 7: Potential therapies of Cold Atmospheric Pressure Plasma (CAP) promoting wound healing and reducing scar formation during wound repair. (ROS, reactive oxygen species; RNS, reactive nitrogen species).

\section{Roles and Future Prospect of Cap in Medical Practice}

There were no significant differences in any of the evaluated items between the groups treated with CAP, ointment, and bFGF. Steroids are often used as an anti-inflammatory drug for wounds without infection, including skin troubles after $\mathrm{FXCO}_{2}$ laser treatment. We observed an improvement in redness, compared with the control group, in the group of irradiated regions treated with ointment containing steroids, confirming its anti-inflammatory action. However, although the incidence is low, steroidal drug use carries a risk of developing adverse reactions, including steroid acne, steroid rosacea, peristome dermatitis, excessive hair growth, steroid peliosis, skin atrophy, infection disease, contact dermatitis, and pigmentation. Caution is therefore needed to avoid severe burning or skin injury to the face. In Japan, the use of bFGF is covered by national health insurance for the treatment of compression gangrene or skin ulcers, so it is often applied in clinical practice. However, issues with bFGF include its high cost (about US $\$ 83.60$ for a $5 \mathrm{~mL}$ bottle), the need for cold storage, the short effective time for consumption (within 2 weeks), and its lack of antibacterial effects. Steroids and bFGF preparation cannot be shared with other patients after opening their containers, which means that waste of medical resources often becomes an issue. In this study, the plasma treatment showed various benefits: anti-inflammatory action equivalent to that of steroid ointment, the capability for continuous radiation for each patient, and no side effects. Plasma treatment is therefore considered to be effective for post-fractional laser treatment, not just for general injuries. Since medical plasma for skin treatment is simple and safe, and there is abundant evidence of its efficacy, it is considered a promising treatment, with further development expected in the future.

\section{Limitations}

Our study had three limitations. First, the number of subjects was small, and the follow-up period was short. Second, the definition of the required number of subjects for data verification may not have been appropriate because there have been no similar evaluation systems. Third, the treatments were administered to normal tissues of healthy subjects, and we did not conduct any invasive examinations, such as harvesting sample tissues. This meant more objective evaluations, including cellular and molecular evaluations, were not available. To confirm our conclusions, a multiinstitutional large-scale randomized controlled trial, including molecular analysis, is necessary.

\section{Conclusion}

Our randomized controlled trial showed the clinical efficacy of CAP as a new energy device for skin activation. The CAP treatment 
by applying plasma jet resulted in anti-inflammatory action, with efficacy equivalent to that of conventional therapies. Our findings provide confirmatory evidence of the effectiveness and safety of CAP and may assist the future development of medical plasma therapy.

\section{References}

1. Maiman TH (1960) Stimulated optical radiation in ruby. Nature 187: 493-494.

2. Anderson RR, Parrish JA (1983) Selective photothermolysis: Precise microsurgery by selective absorption of pulsed radiation. Science 220(4596): 524-527.

3. Manstein D, Herron GS, Sink RK, Tanner H, Anderson RR (2004) Fractional photothermolysis: A new concept for cutaneous remodeling using microscopic patterns of thermal injury. Lasers surg and med 34(5): 426-438.

4. Narins DJ, Narins RS (2003) Non-surgical radiofrequency facelift. J drugs Dermatol 2(5): 495-500.

5. Gliklich RE, White WM, Slayton MH, Barthe PG, Makin IR (2007) Clinical pilot study of intense ultrasound therapy to deep dermal facial skin and subcutaneous tissues. Arch facial plast surg 9(2): 88-95.

6. Manstein D, Laubach H, Watanabe K, Farinelli W, Zurakowski D, et al. (2008) Selective cryolysis: A novel method of non-invasive fat removal. Lasers Surg Med 40(9): 595-604.

7. Laroussi M (1996) Sterilization of contaminated matter with an atmospheric pressure plasma. IEEE Trans Plasma Sci 24(3): 1188-1191.

8. Ulrich C, Kluschke F, Patzelt A, Vandersee S, Czaika VA, et al. (2015) Clinical use of cold atmospheric pressure argon plasma in chronic leg ulcers: A pilot study. J wound care 24(5): 196,198-200, 202-193.

9. Metelmann H-R, Vu TT, Do HT, Binh Le TN, Hoang TH A, et al. (2013) Scar formation of laser skin lesions after Cold Atmospheric Pressure Plasma (CAP) treatment: A clinical long-term observation. Clin Plasma Med 1(1): 30-35.

10. Vandersee S, Richter H, Lademann J, Beyer M, Kramer A, et al. (2014) Laser scanning microscopy as a means to assess the augmentation of tissue repair by exposition of wounds to tissue tolerable plasma. Laser Phy Letters 11(11): 115701.

11. Weltmann KD, Kindel E, Brandenburg R, Meyer C, Bussiahn R, et al. (2009) Atmospheric pressure plasma jet for medical therapy: Plasma parameters and risk estimation. Contri plasma phy 49(9): 631-640.

12. Clementoni MT, Lavagno R, Catenacci M, Kantor R, Mariotto G, et al. (2011) 3D in vivo optical skin imaging for intense pulsed light and fractional ablative resurfacing of photodamaged skin. Facial plast surg clin North Am 19(4): 737-757.

13. Matias AR, Ferreira M, Costa P, Neto P (2015) Skin colour, skin redness and melanin biometric measurements: comparison study between Antera((R)) 3D, Mexameter((R)) and Colorimeter((R)). Skin Res Technol 21(3): 346-362.

14. Holzer W (1947) Physical medicine in diagnostics and therapy. Maudrich.

15. Bekeschus S, Schmidt A, Weltmann KD, von Woedtke T (2016) The plasma jet kINPen-a powerful tool for wound healing. Clini Plasma Med 4(1): 19-28.

16. Napp J, Daeschlein G, Napp M, von Podewils S, Gümbel D, et al. (2015) On the history of plasma treatment and comparison of microbiostatic efficacy of a historical high-frequency plasma device with two modern devices. GMS hyg infect control 10.

17. Daeschlein G, Scholz S, Ahmed R, von Woedtke T, Haase H, et al. (2012) Skin decontamination by low-temperature atmospheric pressure plasma jet and dielectric barrier discharge plasma. J hosp infect 81(3): 177-183.

18. Guo S, Dipietro LA (2010) Factors affecting wound healing. J Dent Res 89(3): 219-229.

19. Gurtner GC, Werner S, Barrandon Y, Longaker MT (2008) Wound repair and regeneration. Nature 453: 314-321.

20. Murad F (2004) Discovery of some of the biological effects of nitric oxide and its role in cell signaling. Biosci Rep 24(4-5): 452-474.

21. Hirata T, Kishimoto T, Tsutsui C, Kanai T, Mori A (2013) Healing burns using atmospheric pressure plasma irradiation. Japan J App Phy 53(1): 010302.
ISSN: 2574-1241

DOI: 10.26717/BJSTR.2019.21.003532

Akio Nishijima. Biomed J Sci \& Tech Res

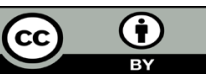

This work is licensed under Creative Commons Attribution 4.0 License

Submission Link: https://biomedres.us/submit-manuscript.php

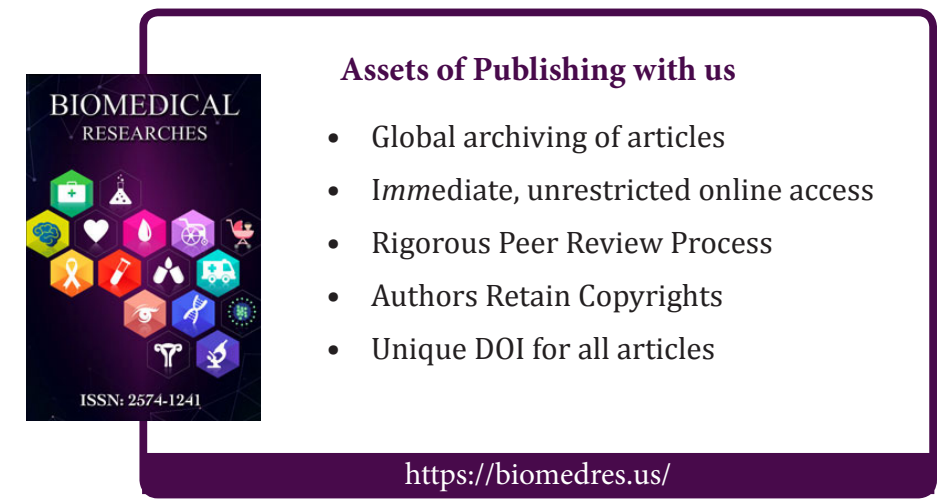

